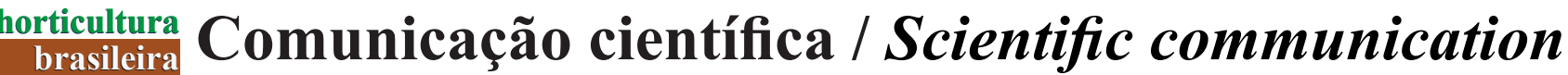

OLIVEIRA FA; MEDEIROS JF; LINHARES PSF; ALVES RC; MEDEIROS AMA; OLIVEIRA MKT. 2014 Produção de mudas de pimenta fertirrigadas com diferentes soluções nutritivas. Horticultura Brasileira 32: 458-463. DOI - http://dx.doi.org/10.1590/S0102-053620140000400014

\section{Produção de mudas de pimenta fertirrigadas com diferentes soluções nutritivas}

\section{Francisco A Oliveira; José F Medeiros; Paulo SF Linhares; Rita C Alves; Arthur MA Medeiros; Mychelle KT Oliveira}

UFERSA, Av. Francisco Mota 572, Costa e Silva, 59625-900 Mossoró-RN; thikaoamigao@ufersa.edu.br; jfmedeir@ufersa.edu.br; paulo.catole@hotmail.com; cassiaagro-23@hotmail.com; arthur_manoel@hotmail.com; mkto10@hotmail.com

\section{RESUMO}

A produção de mudas é uma das etapas mais importantes na produção de hortaliças, principalmente de hortaliças fruto. Este trabalho foi desenvolvido com o objetivo de avaliar a produção de mudas de pimenta fertirrigadas com diferentes soluções nutritivas, em substrato de fibra de coco. Utilizou-se o delineamento inteiramente casualizado, em esquema fatorial $4 \times 8$, com quatro repetições. $O$ primeiro fator foi composto por quatro soluções nutritivas $(0,50,75$ e 100\%) aplicadas via fertirrigação, e o segundo fator foi composto por oito cultivares de pimentas (C1: pimenta Vulcão, C2: pimenta Malagueta, C3: pimenta Tekila, C4: pimenta Ouro, C5: pimenta Doce Comprida, C6: pimenta Cayenne Dedo-de-moça, C7: pimenta Salar e C8: pimenta de cheiro Luna). As mudas foram avaliadas quanto aos parâmetros de desenvolvimento: número de folhas, área foliar, diâmetro do colo, comprimento da raiz principal, altura de plântula e massa seca total. Todas as variáveis foram afetadas pelas concentrações iônicas das soluções nutritivas em todas as cultivares. A concentração de nutrientes na solução de fertirrigação deve ser determinada de acordo com cada cultivar. Para a produção de mudas em sistema floating recomenda-se solução nutritiva com concentração variando de 50$90 \%$ para as cultivares $\mathrm{C} 1, \mathrm{C} 2, \mathrm{C} 3, \mathrm{C} 4$ e $\mathrm{C} 7$; e de $70-90 \%$ para as cultivares $\mathrm{C} 5, \mathrm{C} 6$ e $\mathrm{C} 8$.

Palavras chave: Capsicum frutescens, nutrição de plantas, fertirrigação.

\begin{abstract}
Pepper seedlings production fertigated with various nutrient solutions

Seedling production is one of the most important steps in the production of vegetables, mainly for fruit vegetables. We evaluated the production of pepper seedlings in coconut fiber substrate using fertigation. We used a completely randomized design in a factorial scheme $4 \times 8$ with four replications. The first factor was composed of four nutrient solutions applied through fertigation, and the second factor comprised eight peppers cultivars (C1: Vulcão pepper, $\mathrm{C} 2$ : Malagueta pepper, C3: Tekila pepper, C4: Ouro pepper, C5: Doce Comprida pepper, C6: Cayenne Dedo-de-moça pepper, C7: Salar pepper and C8: Luna smell pepper). The seedlings were evaluated for the following development parameters: number of leaves, leaf area, stem diameter, main root length, height and total dry mass. All variables were affected by the ionic concentrations of nutrient solutions in all cultivars. The concentration of nutrients in fertigation solution should be determined according to the cultivar. A concentration ranging from 50 to $90 \%$ is recommended for the cultivars C1, C2, C3, C4 and C7; and from 70 to $90 \%$ for the cultivars $\mathrm{C} 5, \mathrm{C} 6$ and $\mathrm{C} 8$ for production of seedlings in floating system nutrient solution.
\end{abstract}

Keywords: Capsicum frutescens, plants nutrition, fertigation.

(Recebido para publicação em 3 de maio de 2013; aceito em 9 de agosto de 2014)

(Received on May 3, 2013; accepted on August 9, 2014)

\begin{abstract}
A s pimenteiras pertencem à família Solanaceae, gênero Capsicum. São caracterizadas agronomicamente como cultura olerícola, e as principais espécies cultivadas no Brasil são Capsicum frutescens (pimenta malagueta), $C$. baccatum (dedo-de-moça), C. chinese (de cheiro), C. praetermissum, $C$. аппиит (jalapeño) (Filgueira, 2008).

As pimenteiras se destacam como importantes produtos do agronegócio brasileiro, e os principais estados produtores são Minas Gerais, Goiás, São Paulo, Ceará e Rio Grande do Sul. A região Nordeste apresenta potencial para
\end{abstract}

a produção desta hortaliça, principalmente pelas condições edafoclimáticas favoráveis. No entanto, ainda são escassos estudos sobre esta cultura na região, a começar pela produção de mudas, que é uma das principais fases na produção de hortaliças (Campanharo et al., 2006).

$\mathrm{O}$ uso de substrato na produção de mudas vem aumentando consideravelmente pelos produtores de hortaliças e várias pesquisas já foram desenvolvidas buscando o desenvolvimento de substratos alternativos (Lima et al., 2009; Rodrigues et al., 2010a; Costa et al., 2011).

Dentre os materiais utilizados no preparo de substratos, o pó de coco pode ser utilizado no estágio verde ou seco e apresenta características desejáveis para um bom substrato, tais como alta retenção de umidade, resistência à degradação, uniformidade, ser livre de patógenos e ervas daninhas (Oliveira $e t$ al., 2008, 2009; Sampaio et al., 2008).

O pó de coco é quimicamente inerte e apresenta quantidades de nutrientes não satisfatórias para o desenvolvimento das plântulas. Dessa forma, torna-se imprescindível o fornecimento de nutrientes via fertirrigação para atender às necessidades nutricionais das plân- 
tulas (Braga et al., 2002; Moreira et al., 2010), no entanto, recomendações de soluções nutritivas para a produção de mudas neste substrato são escassas.

Em trabalho desenvolvido com mudas de pimenta malagueta, Pagliarini et al. (2011) utilizaram soluções de fertirrigação variando de 0 a $40 \mathrm{~mL} / \mathrm{L}$ de uma solução padrão de NPK (10-10-10) e obtiveram maior desenvolvimento com a dose de 25 mL/L. Ramos et al. (2012) avaliaram a produção de mudas de melancia em fibra de coco e concluíram que as combinações de pó de coco associado à solução nutritiva de Hoagland \& Arnon a 50, 75 e 100\% mostraram-se promissoras para a formação de mudas de melancia, em relação ao crescimento e status nutricional.

O sistema Floating tem sido utilizado na produção de mudas de várias hortaliças, como pimentão (Verdial et al., 1998), tomate (Rodrigues et al., 2010b) e alface (Hamasaki et al., 2002). Além da forma de aplicação, é importante conhecer a concentração de nutrientes ideal para cada espécie na solução nutritiva.

Este trabalho foi desenvolvido com o objetivo de avaliar a qualidade de mudas de cultivares de pimentas em função da concentração de nutrientes na solução nutritiva.

\section{MATERIAL E MÉTODOS}

O experimento foi conduzido de fevereiro a março de 2012, em casa de vegetação da Universidade Federal Rural do Semi-Árido, localizada no município de Mossoró-RN (5 ${ }^{\circ} 11$ '31' $\mathrm{S}$, $37^{\circ} 20^{\prime} 40^{\prime}$ 'O, altitude $18 \mathrm{~m}$ ).

A casa de vegetação utilizada tem 7 $\mathrm{m}$ de largura com $18 \mathrm{~m}$ de comprimento e estrutura de aço galvanizado. As paredes laterais e frontais são confeccionadas com tela negra (sombrite) com 50\% de sombreamento e têm cobertura em arco tipo túnel feita com filme agrícola com polietileno de baixa densidade e transparente, com $150 \mu \mathrm{m}$ de espessura e tratamento anti-ultravioleta.

O delineamento experimental utilizado foi inteiramente ao acaso, com os tratamentos distribuídos em esquema fatorial 4x8 (quatro soluções nutritivas e oito cultivares de pimenta), com quatro repetições, sendo a unidade experimental representada por uma população de 16 mudas.

Os tratamentos resultaram da combinação de quatro concentrações de nutrientes na solução nutritiva $[0,50$, 75 e $100 \%$ da dose recomendada conforme Castellane \& Araújo (1994) para a cultura do pimentão] e oito cultivares de pimenta $(\mathrm{C} 1$ : pimenta Vulcão, C2: pimenta Malagueta, C3: pimenta Tekila, C4: pimenta Ouro, C5: pimenta Doce Comprida, C6: pimenta Cayenne Dedo-de-moça, C7: pimenta Salar e C8: pimenta de cheiro Luna). Utilizou-se pó de coco (Golden Mix Granulado) como substrato, composto a partir de $100 \%$ de fibra de coco, de textura fina, sem adubação de base. Utilizaram-se bandejas plásticas com 200 células, com formato piramidal, nas quais foram semeadas quatro sementes por célula, realizando-se o desbaste cinco dias após a emergência, deixando-se em cada célula a plântula mais vigorosa.

No período entre a semeadura e o desbaste, as irrigações foram realizadas utilizando um regador manual, aplicando-se apenas água, e após o desbaste iniciou-se a aplicação das soluções nutritivas de acordo com cada tratamento.

No preparo das soluções foi utilizada água proveniente do sistema de abastecimento do campus central da UFERSA, apresentando as características: $\mathrm{pH}=$ 8,3; $\mathrm{CE}=0,5 \mathrm{dS} / \mathrm{m} ; \mathrm{Ca}=2,0 ; \mathrm{Mg}=0,9$; $\mathrm{Na}=2,87 ; \mathrm{K}=0,4 ; \mathrm{HCO}_{3}=4,0 ; \mathrm{CO}_{3}=$ 0,$2 ; \mathrm{Cl}=1,8\left(\mathrm{mmol}_{\mathrm{c}} / \mathrm{L}\right)$.

A solução padrão (100\%) apresentava macronutrientes na concentração: $\mathrm{N}=152 ; \mathrm{P}=29, \mathrm{~K}=245 ; \mathrm{Ca}=20$ e $\mathrm{Mg}=$ $32(\mathrm{mg} / \mathrm{L})$, recomendada para a cultura do pimentão (Castelhane \& Araújo, 1994). Como fonte de micronutrientes, utilizou-se Quelatec ${ }^{\circledR}$ (mistura sólida de EDTA-chelated nutrients, contendo 0,28\% Cu, 7,5\% Fe, 3,5\% Mn, 0,7\% Zn, $0,65 \%$ B e $0,3 \%$ Mo), aplicando-se a dosagem de 6 g de Quelatec ${ }^{\circledR}$ para cada 100 L de solução.

Após o preparo das soluções determinou-se suas respectivas condutividades elétricas, obtendo-se os valores: 0,$56 ; 1,31 ; 1,74$ e $2,55 \mathrm{dS} / \mathrm{m}$, para as concentração de $0,50,75$ e $100 \%$ da dose recomendada, respectivamente.
O sistema floating foi instalado sobre bancada de madeira com dimensões de $5 \times 1 \mathrm{~m}$, sobre cavaletes em altura de $1 \mathrm{~m}$. A parte superior da bancada foi dividida em 5 partes com dimensões de 80x80 cm utilizando pedaços de madeira (caibros). Cada parte foi recoberta com lona plástica para formar uma micro-piscina com capacidade para acondicionar duas bandejas.

As bandejas permaneceram em lâmina de água de $1 \mathrm{~cm}$ até a retirada das mudas. Diariamente era realizada a reposição da solução nutritiva em todos os tratamentos, aplicando o volume suficiente para manter a solução nutritiva com lâmina de $1 \mathrm{~cm}$.

As mudas foram coletadas aos 36 dias após a semeadura, analisando 10 mudas de cada tratamento para serem avaliadas quanto às características: número de folhas completamente expandidas (NF), área foliar (AF), diâmetro do colo (DC, medida à altura de $0,5 \mathrm{~cm}$ da superfície do torrão), comprimento da raiz principal (CRP), altura da plântula (ALT) e massa seca total (MST).

Os dados obtidos foram submetidos à análise de variância e as médias resultantes das cultivares foram comparadas entre si aplicando-se o teste Scott-Knott ao nível de $5 \%$ de probabilidade. Os dados referentes ao efeito da concentração iônica na solução nutritiva foram submetidos à análise de regressão. Os resultados foram analisados no sistema computacional de análise de variância, Sisvar 5.3 (Ferreira, 2011).

\section{RESULTADOS E DISCUSSÃO}

Houve efeito significativo da interação entre os fatores soluções nutritivas $\mathrm{x}$ cultivares ao nível de significância de $1 \%$ de probabilidade para número de folhas (NF), diâmetro do colo (DC), altura (ALT), área foliar (AF) e massa seca total (MST), e ao nível de 5\% de probabilidade para comprimento da raiz principal (CRP). Com relação ao efeito dos fatores isolados, verificou-se resposta significativa para os fatores solução nutritiva e para cultivares em nível de $1 \%$ de probabilidade (Tabela 1 ).

Mudas com maior número de folhas foram observadas nas cultivares $\mathrm{C} 1$ 
Tabela 1. Valores médios para número de folhas (NF), área foliar (AF), diâmetro do colo (DC), comprimento da raiz principal (CRP), altura (ALT) e massa seca total (MST), em mudas de pimentas fertirrigadas com diferentes soluções nutritivas [average values of number of leaves (NF), leaf area (AF), stem diameter (DC), main root length (CRP), height (ALT) and total dry matter (MST) of pepper seedlings fertirrigated with various nutritive solutions]. Mossoró, UFERSA, 2012.

\begin{tabular}{lcccccc}
\hline Cultivares & NF & $\mathbf{A F}\left(\mathbf{c m}^{2}\right)$ & $\mathbf{D C}(\mathbf{m m})$ & $\mathbf{C R P}(\mathbf{c m})$ & ALT $(\mathbf{c m})$ & $\mathbf{M S T}(\mathbf{g})$ \\
\hline Pimenta Vulcão & $7,29 \mathrm{a}^{*}$ & $75,78 \mathrm{~b}$ & $1,30 \mathrm{c}$ & $8,51 \mathrm{a}$ & $6,52 \mathrm{c}$ & $0,36 \mathrm{~b}$ \\
Pimenta Malagueta & $5,53 \mathrm{~b}$ & $80,69 \mathrm{~b}$ & $1,04 \mathrm{~d}$ & $9,49 \mathrm{a}$ & $3,74 \mathrm{~d}$ & $0,26 \mathrm{c}$ \\
Pimenta Tekila & $4,72 \mathrm{c}$ & $37,07 \mathrm{c}$ & $1,06 \mathrm{~d}$ & $8,21 \mathrm{a}$ & $2,67 \mathrm{e}$ & $0,13 \mathrm{~d}$ \\
Pimenta Ouro & $4,29 \mathrm{~d}$ & $25,09 \mathrm{~d}$ & $0,86 \mathrm{f}$ & $7,02 \mathrm{~b}$ & $2,21 \mathrm{e}$ & $0,10 \mathrm{~d}$ \\
Pimenta Doce comprida & $5,66 \mathrm{~b}$ & $96,72 \mathrm{a}$ & $1,59 \mathrm{a}$ & $7,49 \mathrm{~b}$ & $9,31 \mathrm{a}$ & $0,59 \mathrm{a}$ \\
Pimenta Cayenne Dedo-de-moça & $7,26 \mathrm{a}$ & $82,50 \mathrm{~b}$ & $1,39 \mathrm{~b}$ & $8,44 \mathrm{a}$ & $7,96 \mathrm{~b}$ & $0,38 \mathrm{~b}$ \\
Pimenta Salar & $4,66 \mathrm{c}$ & $25,75 \mathrm{~d}$ & $0,94 \mathrm{e}$ & $8,51 \mathrm{a}$ & $2,36 \mathrm{e}$ & $0,11 \mathrm{~d}$ \\
Pimenta de cheiro Luna & $4,73 \mathrm{c}$ & $21,15 \mathrm{~d}$ & $1,03 \mathrm{~d}$ & $7,28 \mathrm{~b}$ & $2,34 \mathrm{e}$ & $0,11 \mathrm{~d}$ \\
\hline Soluções (S) & $* *$ & $* *$ & $* *$ & $* *$ & $* *$ & $* *$ \\
Cultivares (C) & $* *$ & $* *$ & $* *$ & $* *$ & $* *$ & $* *$ \\
Interação SxC & $* *$ & $* *$ & $* *$ & $*$ & $* *$ & $* *$ \\
\hline CV (\%) & 8,76 & 13,45 & 7,62 & 14,57 & 12,51 & 11,72 \\
\hline
\end{tabular}

*Médias seguidas pela mesma letra na coluna não diferem entre si, pelo teste de Scott-Knott, 5\%; ${ }^{*},{ }^{*}, * *$ não significativo, significativos a 5 e $1 \%$ de probabilidade, respectivamente (means followed by the same letter in the column do not differ significantly, according to ScottKnott test, $5 \%$; ${ }^{\text {ns }}{ }^{*}, * *$ nonsignificant, significant at 5 and $1 \%$ probability).

(7,29 folhas/muda) e C6 (7,26 folhas/ muda), as quais não diferiram estatisticamente entre si. O menor NF foi observado na cultivar C4 (4,29 folhas/ muda) (Tabela 1).

O número de folhas (NF) aumentou em resposta ao aumento da concentração de nutrientes na solução nutritiva até determinada concentração, a partir da qual ocorreu decréscimo na emissão de novas folhas. A concentração que proporcionou o máximo NF variou de acordo com cada cultivar. Os resultados se ajustaram melhor a equações quadráticas para todas as cultivares estudadas; no entanto, a concentração que apresentou o máximo NF variou de acordo com a cultivar analisada. Os maiores valores para NF ocorreram nas concentrações de $75,77,60,66,92,75,65$ e $67 \%$ da dose recomendada, respectivamente para as cultivares $\mathrm{C} 1, \mathrm{C} 2, \mathrm{C} 3, \mathrm{C} 4, \mathrm{C} 5, \mathrm{C} 6, \mathrm{C} 7$ e C8. Utilizando-se essas concentrações de solução nutritiva, estimou-se maior NF para C1 e C6, com 9,3 e 9,5 folhas/ muda, respectivamente, equivalente ao ganho percentual de aproximadamente $263 \%$ para $\mathrm{C} 1$ e $495 \%$ para $\mathrm{C} 6$, em relação às mudas irrigadas apenas com água (Tabela 2).

Resultados semelhantes foram encontrados por outros autores trabalhando com adubação na produção de mudas de outras espécies da mesma família botânica, a exemplos de berinjela
(Moreira et al., 2010) e tomate (Silveira et al., 2002), nas quais o menor número de folhas ocorreu em mudas que não receberam fertirrigação $(\mathrm{S} 1)$.

Para área foliar, verificou-se que os maiores valores ocorreram na cultivar C5 $\left(96,72 \mathrm{~cm}^{2}\right)$, seguida pelas cultivares $\mathrm{C} 1, \mathrm{C} 2$ e C6, que não diferiam entre si estatisticamente, e apresentaram AF média variando de 75,78 a $82,50 \mathrm{~cm}^{2}$. Os menores valores foram obtidos nas cultivares $\mathrm{C} 4, \mathrm{C} 7$ e $\mathrm{C} 8$, com $\mathrm{AF}$ variando de 21,15 a $25,75 \mathrm{~cm}^{2}$ (Tabela 1).

A área foliar é uma das variáveis mais importantes a ser avaliada, tendo em vista que quanto maior a $\mathrm{AF}$, maior a área disponível para captação de energia e realização de fotossíntese pelas plantas, convertendo energia luminosa em energia química, essencial para seu crescimento e desenvolvimento (Taiz \& Zeiger, 2009).

Quanto ao efeito das soluções nutritivas sobre a AF, foram observados resultados semelhantes aos ocorridos para $\mathrm{NF}$, com os dados apresentando melhor ajuste à equação quadrática, para todas as cultivares estudadas. A partir das equações de regressão obtidas, pôde-se agrupar as cultivares $\mathrm{C} 1, \mathrm{C} 2, \mathrm{C} 3, \mathrm{C} 4, \mathrm{C} 7$ e $\mathrm{C} 8$, que apresentaram maiores valores de AF para concentração variando de 52 a $63 \%$, enquanto que as cultivares $\mathrm{C} 5 \mathrm{e}$ $\mathrm{C} 6$ apresentaram maior requerimento de nutrientes e apresentaram maiores valores para concentrações de 78 e $73 \%$, respectivamente (Tabela 2). Vale ressaltar que apesar da cultivar C5 apresentar maior exigência nutricional que as demais cultivares, a $\mathrm{C} 5$ apresentou maior desenvolvimento foliar $\left(136,34 \mathrm{~cm}^{2}\right)$.

Pagliarini et al. (2011) avaliaram o efeito de doses crescentes de fertilizante líquido na produção de mudas de pimenta malagueta e verificaram resposta quadrática para número de folhas e área foliar, semelhantes aos resultados obtidos no presente trabalho.

O diâmetro do colo também diferiu significativamente entre as cultivares, com maior valor ocorrendo para a cultivar C5 $(1,59 \mathrm{~mm})$, seguida pela C6 $(1,39 \mathrm{~mm})$, enquanto os menores valores ocorreram em mudas da cultivar $\mathrm{C} 4$ $(0,86 \mathrm{~mm})$ (Tabela 1).

Verificou-se resposta variada das cultivares ao aumento na concentração de nutrientes na solução nutritiva para o diâmetro do colo (DC), sendo observado comportamento quadrático para todas as cultivares estudadas. De acordo com as equações de regressão ajustadas verificou-se que as cultivares $\mathrm{C} 1, \mathrm{C} 2$, $\mathrm{C} 3, \mathrm{C} 4, \mathrm{C} 7$ e $\mathrm{C} 8$ apresentaram maiores valores de $\mathrm{DC}$ ocorrendo em concentrações variando de $54-70 \%$, enquanto as cultivares C5 e C6 apresentaram maiores valores para concentrações variando de 76-85\% (Tabela 2). 
Tabela 2. Equações de regressão para as variáveis número de folhas (NF), área foliar (AF), diâmetro do colo (DC), comprimento da raiz principal (CRP), altura (ALT) e massa seca total (MST) de plântulas de pimenta utilizando diferentes soluções nutritivas [regression equations for the variables number of leaves (NF), leaf area (AF), stem diameter (DC), length of the main root (CRP), height (ALT) and total dry matter (MST) of pepper seedlings fertirrigated with various nutritive solutions]. Mossoró, UFERSA, 2012.

\begin{tabular}{|c|c|c|c|c|c|}
\hline \multirow{2}{*}{ Cultivares - } & \multirow{2}{*}{$\begin{array}{l}\text { Equação de regressão } \\
\text { Número de folhas }\end{array}$} & \multirow{2}{*}{$\mathbf{R 2}$} & \multirow{2}{*}{ Cultivares } & \multirow{2}{*}{$\frac{\text { Equação de regressão }}{\text { Área foliar }}$} & \multirow{2}{*}{$\mathbf{R 2}$} \\
\hline & & & & & \\
\hline $\mathrm{C} 1$ & $\mathrm{NF}=2,57+0,18 * * \mathrm{X}-0,0012 * * \mathrm{X}^{2}$ & 0,981 & $\mathrm{C} 1$ & $\mathrm{AF}=4,38+3,97 * * \mathrm{X}-0,034 * * \mathrm{X}^{2}$ & 0,999 \\
\hline $\mathrm{C} 2$ & $\mathrm{NF}=1,64+0,16^{* *} \mathrm{X}-0,0011^{* *} \mathrm{X}^{2}$ & 0,999 & $\mathrm{C} 2$ & $\mathrm{AF}=1,77+4,08^{* *} \mathrm{X}-0,033^{* *} \mathrm{X}^{2}$ & 0,988 \\
\hline $\mathrm{C} 3$ & $\mathrm{NF}=2,13+0,12 * * \mathrm{X}-0,0010 * * \mathrm{X}^{2}$ & 0,999 & $\mathrm{C} 3$ & $\mathrm{AF}=1,61+1,88^{* * \mathrm{X}}-0,015^{* *} \mathrm{X}^{2}$ & 0,932 \\
\hline $\mathrm{C} 4$ & $\mathrm{NF}=1,94+0,12 * * \mathrm{X}-0,00091 * * \mathrm{X}^{2}$ & 0,999 & $\mathrm{C} 4$ & $\mathrm{AF}=3,74+1,47 * * \mathrm{X}-0,014 * * \mathrm{X}^{2}$ & 0,886 \\
\hline $\mathrm{C} 5$ & $\mathrm{NF}=2,50+0,11 * * \mathrm{X}-0,0006^{* *} \mathrm{X}^{2}$ & 0,999 & C5 & $\mathrm{AF}=1,08+3,45^{* *} \mathrm{X}-0,022^{* *} \mathrm{X}^{2}$ & 0,899 \\
\hline $\mathrm{C} 6$ & $\mathrm{NF}=1,59+0,21 * * \mathrm{X}-0,0014 * * \mathrm{X}^{2}$ & 0,997 & C6 & $\mathrm{AF}=1,23+3,22 * * \mathrm{X}-0,022 * * \mathrm{X}^{2}$ & 0,972 \\
\hline $\mathrm{C} 7$ & $\mathrm{NF}=2,13+0,13^{* *} \mathrm{X}-0,0010^{* *} \mathrm{X}^{2}$ & 0,999 & $\mathrm{C} 7$ & $\mathrm{AF}=4,62+1,56^{* *} \mathrm{X}-0,015^{* *} \mathrm{X}^{2}$ & 0,754 \\
\hline $\mathrm{C} 8$ & $\mathrm{NF}=1,99+0,12 * * \mathrm{X}-0,0009 * * \mathrm{X}^{2}$ & 0,968 & $\mathrm{C} 8$ & $\mathrm{AF}=2,96+0,99 * * \mathrm{X}-0,0084 * * \mathrm{X}^{2}$ & 0,977 \\
\hline \multicolumn{3}{|c|}{ Diâmetro do colo } & \multicolumn{3}{|c|}{ Comprimento da raiz principal } \\
\hline $\mathrm{C} 1$ & $\mathrm{DC}=0,56+0,031 * * \mathrm{X}-0,00023 * * \mathrm{X}^{2}$ & 0,983 & $\mathrm{C} 1$ & $\mathrm{CRP}=10,27-0,031 * * \mathrm{X}$ & 0,646 \\
\hline $\mathrm{C} 2$ & $\mathrm{DC}=0,66+0,014 * * X-0,00010^{* *} \mathrm{X}^{2}$ & 0,889 & $\mathrm{C} 2$ & $\mathrm{CRP}=11,48+0,060 * * \mathrm{X}-0,0012 * * \mathrm{X}^{2}$ & 0,992 \\
\hline $\mathrm{C} 3$ & $\mathrm{DC}=0,72+0,016^{* *} \mathrm{X}-0,00013^{* *} \mathrm{X}^{2}$ & 0,855 & $\mathrm{C} 3$ & $\mathrm{CRP}=11,01-0,049^{* *} \mathrm{X}$ & 0,897 \\
\hline $\mathrm{C} 4$ & $\mathrm{DC}=0,71+0,009^{* *} \mathrm{X}-0,00008^{* *} \mathrm{X}^{2}$ & 0,730 & $\mathrm{C} 4$ & $\mathrm{CRP}=11,15-0,055^{* *} \mathrm{X}$ & 0,909 \\
\hline $\mathrm{C} 5$ & $\mathrm{DC}=0,56+0, \mathrm{C}$ & 0,999 & $\mathrm{C} 5$ & $\mathrm{CRP}=9,61-0$, & 0,943 \\
\hline C6 & $\mathrm{DC}=0,68+0,026^{* *} \mathrm{X}-0,00017^{* *} \mathrm{X}^{2}$ & 0,984 & C6 & $\mathrm{CRP}=11,35-0,052 * * \mathrm{X}$ & 0,962 \\
\hline $\mathrm{C} 7$ & $\mathrm{DC}=0,73+0,013^{* *} \mathrm{X}-0,00012^{* *} \mathrm{X}^{2}$ & 0,958 & $\mathrm{C} 7$ & $\mathrm{CRP}=11,10-0,046^{* *} \mathrm{X}$ & 0,889 \\
\hline $\mathrm{C} 8$ & $\mathrm{DC}=0,71+0,0095^{*} \mathrm{X}-0,00005^{*} * \mathrm{X}^{2}$ & 0,801 & & $\mathrm{CRP}=10,72-0,061^{* *} \mathrm{X}$ & 0,955 \\
\hline \multicolumn{3}{|c|}{ Altura das mudas } & \multicolumn{3}{|c|}{ Massa seca total } \\
\hline $\mathrm{C} 1$ & ALT $=1,28+0,28 * * X-0,0023^{* *} \mathrm{X}^{2}$ & 0,998 & $\mathrm{C} 1$ & MST $=0,036+0,018 * * X-0,00015 * * X^{2}$ & 0,964 \\
\hline $\mathrm{C} 2$ & ALT $=1,09+0,15^{* *} \mathrm{X}-0,0012 * * \mathrm{X}^{2}$ & 0,997 & $\mathrm{C} 2$ & MST $=0,036+0,014 * * X-0,00012 * * X^{2}$ & 0,881 \\
\hline $\mathrm{C} 3$ & ALT $=1,11+0,079 * * X-0,00064 * * X^{2}$ & 0,946 & $\mathrm{C} 3$ & MST $=0,042+0,0058^{* * X}-0,00005^{* *} X^{2}$ & 0,788 \\
\hline $\mathrm{C} 4$ & $\mathrm{ALT}=1,20+0,061 * * \mathrm{X}-0,00053 * * \mathrm{X}^{2}$ & 0,872 & $\mathrm{C} 4$ & $\mathrm{MST}=0,042+0,0046 * \mathrm{X}-0,00004 * \mathrm{X}^{2}$ & 0,999 \\
\hline $\mathrm{C} 5$ & $\mathrm{ALT}=0,89+0,29 * * \mathrm{X}-0,0017^{* *} \mathrm{X}^{2}$ & 0,835 & $\mathrm{C} 5$ & MST $=0,027+0,014 * X-0,00008 * X^{2}$ & 0,882 \\
\hline C6 & $\mathrm{ALT}=0,85+0,31 * \mathrm{X}-0,0022 * * \mathrm{X}^{2}$ & 0,916 & C6 & $\mathrm{MST}=0,032+0,013 * * \mathrm{X}-0,00009 * * \mathrm{X}^{2}$ & 0,864 \\
\hline $\mathrm{C} 7$ & $\mathrm{ALT}=1,18+0,073 * \mathrm{X}-0,00064 * * \mathrm{X}^{2}$ & 0,965 & $\mathrm{C} 7$ & $\mathrm{MST}=0,053+0,0042 * \mathrm{X}-0,00004 * \mathrm{X}^{2}$ & 0,871 \\
\hline $\mathrm{C} 8$ & ALT $=1,18+0,052 * X-0,00039 * * X^{2}$ & 0,938 & $\mathrm{C} 8$ & MST $=0,043+0,0028 * X-0,00002 * X^{2}$ & 0,947 \\
\hline
\end{tabular}

*,**significativos a 5 e $1 \%$ de probabilidade pelo teste t; (C1: pimenta Vulcão, C2: pimenta Malagueta, C3: pimenta Tekila, C4: pimenta Ouro, C5: pimenta Doce comprida, C6: pimenta Cayenne Dedo-de-moça, C7: pimenta Salar e C8: pimenta de cheiro Luna) [*,**significant at 5 and $1 \%$ probability by t test; (C1: Vulcão pepper, C2: Malagueta pepper, C3: Tekila pepper, C4: Ouro pepper, C5: Doce comprida pepper, C6: Cayenne Dedo-de-moça pepper, C7: Salar pepper and C8 Luna smell pepper)].

De acordo com Souza et al. (2013) o comprimento da parte aérea, combinado com o diâmetro do colo, constitui um dos mais importantes caracteres morfológicos para se estimar o crescimento das mudas após o plantio definitivo no campo. Neste contexto, mudas de pimenteiras, produzidas com concentração de solução nutritiva acima de $70 \%$ da solução padrão, apresentam maior capacidade de desenvolvimento após o transplantio e, consequentemente, plantas mais vigorosas e mais produtivas.

O comprimento da raiz principal (CRP) também diferiu entre as cultivares estudadas e apresentou menor variação em relação às demais variáveis, permitindo-se obter dois grupos, o primeiro com CRP variando de 8,21 a 9,49 $\mathrm{cm}$ para as cultivares $\mathrm{C} 1, \mathrm{C} 2, \mathrm{C} 3, \mathrm{C} 6 \mathrm{e}$ $\mathrm{C} 7$, e o segundo grupo com as cultivares $\mathrm{C} 4, \mathrm{C} 5$ e $\mathrm{C} 8$, com CRP variando de 7,02 a 7,49 $\mathrm{cm}$ (Tabela 1).

O CRP foi reduzido significativamente e linearmente pelo aumento da concentração de nutrientes na solução nutritiva para as cultivares estudadas $\mathrm{C} 1, \mathrm{C} 3, \mathrm{C} 4, \mathrm{C} 5$, ,C6, C7 e C8, de forma que os maiores valores de CRP foram obtidos nas mudas produzidas na ausência de solução nutritiva. Para a cultivar C2 também houve redução no CRP nas maiores concentrações. Entretanto, concentração de até $50 \%$ da dose recomendada não afetou negativamente o CRP (Tabela 2).

Estes resultados divergem dos obtidos por Pagliarini et al. (2011), que não observaram efeito da fertirrigação sobre o comprimento da raiz. No entanto, vale salientar que esses autores aplicaram os nutrientes via adubação foliar.

Outro provável motivo para a redução no comprimento da raiz com o aumento da concentração de nutrientes pode estar relacionado ao aumento da salinidade da solução, conforme observação feita por Nascimento et al. (2011), que verificaram redução no CRP em mudas de pimentão em resposta ao estresse salino.

A altura das mudas apresentou grande variabilidade entre as cultivares, com maior ALT observada na cultivar C5 $(9,31 \mathrm{~cm})$, enquanto as menores mudas foram obtidas nas cultivares $\mathrm{C} 3, \mathrm{C} 4$, $\mathrm{C} 7$ e $\mathrm{C} 8$, com ALT média variando de 
2,21 a 2,67 cm, não diferindo entre si estatisticamente (Tabela 1).

$\mathrm{Na}$ literatura não existe recomendação sobre a altura ideal para transplantio de mudas de pimenta. No entanto, ao considerar que mudas de pimentão com idade de 30 a 45 dias devem ter altura de 7 a $8 \mathrm{~cm}$ (Filgueira, 2008), percebe-se que no período de avaliação, as mudas produzidas no presente trabalho estariam aptas para serem transplantadas. Vale ressaltar que as mudas foram avaliadas aos 36 dias após a semeadura, e que poderiam ser mantidas por mais tempo sob fertirrigação para apresentarem maior desenvolvimento.

Também foram observadas respostas quadráticas das cultivares para a altura (ALT) das mudas em resposta ao aumento da concentração de nutrientes na solução nutritiva. Para as cultivares $\mathrm{C} 1, \mathrm{C} 2, \mathrm{C} 3, \mathrm{C} 4, \mathrm{C} 6, \mathrm{C} 7$ e C8 os maiores valores foram estimados para concentrações entre $60-70 \%$, enquanto na cultivar C5 obteve-se o maior valor na concentração de $85 \%$, sendo as mudas mais altas observadas nas cultivares $\mathrm{C} 1$ $(9,8 \mathrm{~cm}), \mathrm{C} 5(13,3 \mathrm{~cm})$ e C6 $(11,8 \mathrm{~cm})$ (Tabela 2).

Resultados semelhantes foram obtidos por Pagliarini et al. (2011) trabalhando com fertirrigação em mudas de pimenta malagueta, que também observaram resposta quadrática para altura de mudas com o aumento da concentração de nutrientes na solução nutritiva.

Quanto ao acúmulo de biomassa, apenas a cultivar C5 se destacou das demais, apresentando maior massa seca total $(0,59 \mathrm{~g}$ por muda), seguida pelas cultivares $\mathrm{C} 1$ e C6, com MST de 0,36 e $0,38 \mathrm{~g}$ por muda, respectivamente. Enquanto os menores valores foram observados nas cultivares $\mathrm{C} 3, \mathrm{C} 4, \mathrm{C} 7 \mathrm{e}$ $\mathrm{C} 8$, com MST variando de 0,10-0,13 g/ muda, e não diferindo entre si estatisticamente (Tabela 1).

De modo semelhante ao observado para a maioria das variáveis, a massa seca total (MST) também foi afetada de forma quadrática para todas as cultivares em resposta ao aumento da concentração de nutrientes na solução nutritiva. Para as cultivares $\mathrm{C} 1, \mathrm{C} 2, \mathrm{C} 3, \mathrm{C} 4$ e $\mathrm{C} 7$, os maiores valores ocorreram em concentrações variando de 52 a $60 \%$; enquanto nas cultivares $\mathrm{C} 8$, C6 e $\mathrm{C} 5$ obtiveram-se maiores MST em concentrações entre $69-87 \%$. Considerando as concentrações que proporcionaram maiores valores estimados para acúmulo de massa seca, as cultivares $\mathrm{C} 1$ ( $0,58 \mathrm{~g} /$ muda), $\mathrm{C} 2$ ( 0,44 g/muda), C5 (0,64 g/muda) e C6 (0,50 $\mathrm{g} /$ muda) destacaram-se por apresentar maior MST (Tabela 2).

Em trabalho com substrato de fibra de coco verde e níveis de fertirrigação na produção de mudas de pimentão, Braga et al. (2002) observaram que o uso de fertirrigação favoreceu o desenvolvimento das mudas, aumentando visivelmente o crescimento da parte aérea e antecipando, em pelo menos três dias o tempo de obtenção das mudas.

A massa seca é uma das variáveis mais importantes, e é composta principalmente pelas folhas, e estas constituem uma das principais fontes de fotoassimilados (açúcares, aminoácidos, hormônios, etc.) e nutrientes para adaptação da muda pós-plantio, a qual necessitará de boa reserva de fotoassimilados, que servirão de suprimento de água e nutrientes para as raízes (Bellote \& Silva, 2000).

Analisando o desenvolvimento destas cultivares em todas as variáveis estudadas, percebe-se a importância do manejo diferenciado na produção de mudas, buscando adaptar a programação do setor de produção de mudas de acordo com as características de desenvolvimento para cada cultivar. De acordo com Rodrigues et al. (2010b), a nutrição das mudas é outro fator importante, pois exerce uma influencia marcante no sistema radicular e no estado nutricional das plântulas, afetando profundamente a qualidade das mudas.

Os resultados obtidos no presente trabalho demonstram a importância do fornecimento de nutrientes na produção de mudas produzidas em substrato de fibra de coco, devido, principalmente, à reduzida concentração de nutrientes no substrato, reforçando a recomendação de Oliveira et al. (2009) que a produção de mudas nesse substrato só é viável com o uso de adubação.

No entanto, esses resultados também evidenciam que concentrações muito elevadas podem afetar negativamente o desenvolvimento das mudas, em consequência do estresse salino, estando de acordo com os relatos de Nascimento et al. (2011), os quais trabalhando com pimentão, verificaram que durante a fase de formação das mudas, estas não toleram condutividade elétrica da água de irrigação superior a 2,5 dS/m.

A partir dos resultados obtidos neste trabalho pôde-se constatar que a concentração de nutrientes na solução de fertirrigação deve ser determinada de acordo a cada cultivar. Recomenda-se o uso de soluções nutritivas com concentração de $50-60 \%$ para as cultivares $\mathrm{C} 1, \mathrm{C} 2, \mathrm{C} 3, \mathrm{C} 4$ e C7; e de 70-90\% para as cultivares $\mathrm{C} 5, \mathrm{C} 6$ e $\mathrm{C} 8$, tomando-se como base a dosagem recomendada para a cultura do pimentão em cultivo hidropônico, utilizando fertirrigação em sistema floating.

\section{REFERÊNCIAS}

BELLOTE AFJ; SILVA HD. 2000. Técnicas de amostragem e avaliações nutricionais em plantios de Eucalyptus spp. In: Gonçalves JLM; BENEDETTI V. Nutrição e fertilização florestal. Piracicaba: IPEF, p. 105-133.

BRAGA DO; SOUZA RB; CARRIJO OA; LIMA JL. 2002. Produção de mudas de pimentão em diferentes substratos à base de fibra de coco verde sob fertirrigação Horticultura Brasileira 20: 533-536.

CAMPANHARO M; RODRIGUES JJV; LIRA JUNIOR MAL; ESPINDULA MC; COSTA JVT. 2006. Características físicas de diferentes substratos para produção de mudas de tomateiro. Revista Caatinga 19: 40-145.

CASTELLANE PD; ARAÚJO JAC. 1994. Cultivo sem solo - Hidroponia. Jaboticabal/ FUNEP. 43p.

COSTA E; DURANTE LGY; NAGEL PL; FERREIRA CR; SANTOS A. 2011. Qualidade de mudas de berinjela submetida a diferentes métodos de produção. Revista Ciência Agronômica 42: 1017-1025.

FERREIRADF. 2011. Sisvar: a computer statistical analysis system. Ciência e Agrotecnologia 35: 1039-1042.

FILGUEIRA FAR. 2008. Novo manual de olericultura: Agrotecnologia moderna na produção e comercialização de hortaliças, Viçosa: UFV. 421p.

HAMASAKI RI; BRAZ LT; GRILLI GVG. 2002. Produção e avaliação de mudas de alface no sistema flutuante. Horticultura Brasileira 20: 577-578.

LIMA CJGS; OLIVEIRA FA; MEDEIROS JF; OLIVEIRA MKT; GALVÃO DC. 2009. Avaliação de diferentes bandejas e substratos orgânicos na produção de mudas de tomate cereja. Revista Ciência Agronômica 40: 123-128.

MOREIRA MA; DANTAS FM; BIANCHINI FG; VIÉGAS PRA. 2010. Produção de mudas 
de berinjela com uso de pó de coco. Revista Brasileira de Produtos Agroindustriais 12: 163-170.

NASCIMENTO JAM; CAVALCANTE LF; SANTOS PD; SILVA SA; VIEIRA MS; OLIVEIRA AP. 2011. Efeito da utilização de biofertilizante bovino na produção de mudas de pimentão irrigadas com água salina. Revista Brasileira de Ciências Agrárias 6: 258-264.

OLIVEIRA AB; HERNANDEZ FFF; ASSIS JÚNIOR RN. 2008. Pó de coco verde, uma alternativa de substrato na produção de mudas de berinjela. Revista Ciência Agronômica 39: 39-44.

OLIVEIRA AB; HERNANDEZ FFF; ASSIS JÚNIOR RN. 2009. Absorção de nutrientes em mudas de berinjela cultivadas em pó de coco verde. Revista Caatinga 22: 139-143.

PAGLIARINI MK; GORDIN CRB; SANTOS AM; BRANDÃO NETO JF; BISCARO
GA. 2011. Uso de fertilizante líquido via fertirrigação em mudas de pimenta malagueta. Tecnologia \& Ciência Agropecuária 5: 13-18.

RAMOS ARP; DIAS RCS; ARAGÃO CA; MENDES AMS. 2012. Mudas de melancia produzidas com substrato à base de pó de coco e soluções nutritivas. Horticultura Brasileira 30: 339-344.

RODRIGUES ET; LEAL PAM; COSTA E; PAULA TS; GOMES VA. 2010a. Produção de mudas de tomateiro em diferentes substratos e recipientes em ambiente protegido. Horticultura Brasileira 28: 483-488.

RODRIGUES DS; LEONARDOAFG; NOMURA ES; TACHIBANA L; GARCIA VA; CORREA CF. 2010b. Produção de mudas de tomateiro em sistemas flutuantes com adubos químicos e água residuária de viveiros de piscicultura. Revista Brasileira de Ciências Agrárias 5: 32-35.

SAMPAIO RA; RAMOS SJ; GUILHERME
DO; COSTA CA; FERNANDES LA. 2008. Produção de mudas de tomateiro em substratos contendo fibra de coco e pó de rocha. Horticultura Brasileira 26: 499-503.

SILVEIRA EB; RODRIGUES VJLB; GOMES AMA; MARIANO RLR; MESQUITA JCP. 2002. Pó de coco como substrato para produção de mudas de tomateiro. Horticultura Brasileira 20: 211-216.

SOUZAEGF; BARROS JÚNIOR AP; SILVEIRA LM; SANTOS MG; SILVA EF. 2013. Emergência e desenvolvimento de mudas de tomate IPA 6 em substratos, contendo esterco ovino. Revista Ceres 60: 902-907.

TAIZ L; ZEIGER E. 2009. Plant physiology. 3.ed. Porto Alegre: Artmed. 719p.

VERDIAL MF; IWATA MF; LIMA MS; TESSARIOLI NETO J. 1998. Influência do sistema "floating" no condicionamento do crescimento de mudas de pimentão (Capsicum annuит L.). Scientia Agricola 55: 25-28. 